UDC 517.521

\author{
A.S. Berdyshev ${ }^{1, *}$, A. Hasanov ${ }^{2}$, A.R. Ryskan ${ }^{1}$ \\ ${ }^{1}$ Abai Kazakh National Pedagogical University, Almaty, Kazakhstan; \\ ${ }^{2}$ Institute of mathematics, Uzbek academy of sciences, Tashkent, Uzbekistan; \\ (E-mail: berdyshev@mail.ru, anvarhasanov@yahoo.com,ryskan.a727@gmail.com)
}

\title{
Decomposition formulas for some quadruple hypergeometric series
}

\begin{abstract}
In the present work, the authors obtained operator identities and decomposition formulas for second order Gauss hypergeometric series of four variables into products containing simpler hypergeometric functions. A Choi-Hasanov method based on the inverse pairs of symbolic operators is used. The obtained expansion formulas for the hypergeometric functions of four variables will allow us to study the properties of these functions. These decompositions are used to study the solvability of boundary value problems for degenerate multidimensional partial differential equations.
\end{abstract}

Keywords: Appell hypergeometric function, Lauricella function, Saran function, Quadruple hypergeometric series, Decomposition formulas, Operator identities, Inverse symbolic operators.

\section{Introduction}

A variety of tasks related to almost all of the most significant sections of mathematical physics and answering urgent technical questions are associated with the special functions applying, such as the Bessel, Hermite, Gaussian hypergeometric functions, etc. Thus, for example, Bessel functions are actively used in solving hydrodynamics, radiophysics, acoustics problems of atomic and nuclear physics. There are applications of Bessel functions in problems of elasticity and thermal conductivity theories (determination of stress concentration near faults, plate oscillation) [1]. Many functions used in astronomy are arranged in series of hypergeometric functions [2]. Also, the hypergeometric functions of many complex variables are applicable to the research of analytic continuation problems of Mellin - Barnes type integrals [3], in the superstring theory [4], and in theoretical aspects of algebraic geometry [5].

Generalized hypergeometric functions are used in solving boundary value problems for shell theory equations whose applications are used in mechanical engineering. A.D. Kovalenko developed the application of the theory of generalized hypergeometric functions to determine the stress state in disks, circular plates of alternant thickness and conical shells of rotation according to the equilibrium linear theory [6]. Multiple hypergeometric series are used in research and development of aerospace systems [7]. At the same time, hypergeometric functions of many variables arise in quantum field theory as a solution of Knizhnik-Zamolodchikov equations [5]. In [8-10], the connection of special functions of the hypergeometric type with the actual problems of the theory of representations of Lie algebras and quantum groups is shown, as well as the application of hypergeometric functions and series to applied problems of various fields.

It should be noted that the Riemann functions and the fundamental solutions of degenerate partial differential equations are expressed in terms of multiple hypergeometric functions. Thus, hypergeometric functions are used in solving boundary value problems for degenerate differential equations [11]. In particular, hypergeometric functions are used in [12] to find the fundamental solutions of a fourdimensional degenerate equation of elliptic type, which can be used in solving known boundary value

\footnotetext{
${ }^{*}$ Corresponding author.

E-mail: berdyshev@mail.ru
} 
problems. Also in [13], Appel hypergeometric functions are used to construct a double layer potential theory.

Second order hypergeometric functions of four variables were introduced in [14,15]. For one class of hypergeometric functions of four variables, various properties, such as decomposition formulas, integral representations were obtained in $[16,17]$. However, it should be noted that decompositions into products of simpler hypergeometric functions can be obtained not for all the introduced second order hypergeometric functions of four variables.

In this paper, we obtain decomposition formulas using operator identities for the following quadruple hypergeometric functions:

$$
\begin{aligned}
& F_{1}^{(4)}\left(a_{1}, a_{2}, b ; c_{1}, c_{2}, c_{3}, c_{4} ; x, y, z, t\right)=\sum_{m, n, p, q=0}^{\infty} \frac{\left(a_{1}\right)_{m+n+p}\left(a_{2}\right)_{q}(b)_{m+n+p+q}}{\left(c_{1}\right)_{m}\left(c_{2}\right)_{n}\left(c_{3}\right)_{p}\left(c_{4}\right)_{q}} \frac{x^{m}}{m !} \frac{y^{n}}{n !} \frac{z^{p}}{p !} \frac{t^{q}}{q !}, \\
& F_{3}^{(4)}\left(a_{1}, a_{2}, a_{3}, b ; c_{1}, c_{2}, c_{3}, c_{4} ; x, y, z, t\right)=\sum_{m, n, p, q=0}^{\infty} \frac{\left(a_{1}\right)_{m+n}\left(a_{2}\right)_{p}\left(a_{3}\right)_{q}(b)_{m+n+p+q}}{\left(c_{1}\right)_{m}\left(c_{2}\right)_{n}\left(c_{3}\right)_{p}\left(c_{4}\right)_{q}} \frac{x^{m}}{m !} \frac{y^{n}}{n !} \frac{z^{p}}{p !} \frac{t^{q}}{q !}, \\
& F_{4}^{(4)}\left(a, b, c ; c_{1}, c_{2}, c_{3}, c_{4} ; x, y, z, t\right)=\sum_{m, n, p, q=0}^{\infty} \frac{(a)_{m+n+p+q}(b)_{m+n+q}(c)_{p}}{\left(c_{1}\right)_{m}\left(c_{2}\right)_{n}\left(c_{3}\right)_{p}\left(c_{4}\right)_{q}} \frac{x^{m}}{m !} \frac{y^{n}}{n !} \frac{z^{p}}{p !} \frac{t^{q}}{q !} \\
& F_{5}^{(4)}\left(a_{1}, a_{2}, b_{1}, b_{2} ; c_{1}, c_{2}, c_{3}, c_{4} ; x, y, z, t\right)=\sum_{m, n, p, q=0}^{\infty} \frac{\left(a_{1}\right)_{m+n+p}\left(a_{2}\right)_{q}\left(b_{1}\right)_{m+n}\left(b_{2}\right)_{p+q}}{\left(c_{1}\right)_{m}\left(c_{2}\right)_{n}\left(c_{3}\right)_{p}\left(c_{4}\right)_{q}} \frac{x^{m}}{m !} \frac{y^{n}}{n !} \frac{z^{p}}{p !} \frac{t^{q}}{q !}, \\
& F_{6}^{(4)}\left(a_{1}, a_{2}, b_{1}, b_{2}, b_{3} ; c_{1}, c_{2}, c_{3}, c_{4} ; x, y, z, t\right)=\sum_{m, n, p, q=0}^{\infty} \frac{\left(a_{1}\right)_{m+n+p}\left(a_{2}\right)_{q}\left(b_{1}\right)_{m+q}\left(b_{2}\right)_{n}\left(b_{3}\right)_{p}}{\left(c_{1}\right)_{m}\left(c_{2}\right)_{n}\left(c_{3}\right)_{p}\left(c_{4}\right)_{q}} \frac{x^{m}}{m !} \frac{y^{n}}{n !} \frac{z^{p}}{p !} \frac{t^{q}}{q !} \\
& F_{8}^{(4)}\left(a_{1}, a_{2}, b_{1}, b_{2} ; c_{1}, c_{2}, c_{3}, c_{4} ; x, y, z, t\right)=\sum_{m, n, p, q=0}^{\infty} \frac{\left(a_{1}\right)_{m+n}\left(a_{2}\right)_{p+q}\left(b_{1}\right)_{m+p}\left(b_{2}\right)_{n}\left(b_{3}\right)_{q}}{\left(c_{1}\right)_{m}\left(c_{2}\right)_{n}\left(c_{3}\right)_{p}\left(c_{4}\right)_{q}} \frac{x^{m}}{m !} \frac{y^{n}}{n !} \frac{z^{p}}{p !} \frac{t^{q}}{q !} \\
& F_{11}^{(4)}\left(a_{1}, a_{2}, b ; c_{1}, c_{2}, c_{3} ; x, y, z, t\right)=\sum_{m, n, p, q=0}^{\infty} \frac{\left(a_{1}\right)_{m+n}\left(a_{2}\right)_{p}\left(a_{3}\right)_{q}(b)_{m+n+p+q}}{\left(c_{1}\right)_{m+p}\left(c_{2}\right)_{n}\left(c_{3}\right)_{q}} \frac{x^{m}}{m !} \frac{y^{n}}{n !} \frac{z^{p}}{p !} \frac{t^{q}}{q !}, \\
& F_{13}^{(4)}\left(a_{1}, a_{2}, a_{3}, a_{4}, b ; c_{1}, c_{2}, c_{3} ; x, y, z, t\right)=\sum_{m, n, p, q=0}^{\infty} \frac{\left(a_{1}\right)_{m}\left(a_{2}\right)_{n}\left(a_{3}\right)_{p}\left(a_{4}\right)_{q}(b)_{m+n+p+q}}{\left(c_{1}\right)_{m+n}\left(c_{2}\right)_{p}\left(c_{3}\right)_{q}} \frac{x^{m}}{m !} \frac{y^{n}}{n !} \frac{z^{p}}{p !} \frac{t^{q}}{q !}
\end{aligned}
$$

where $(a)_{n}=\Gamma(a+m) / \Gamma(a)$ is a Pochhammer symbol.

\section{Operator identities}

By means of Burchnall-Chaundy pair of mutually inverse symbolic operators $\nabla_{x, y}(h)$ and $\Delta_{x, y}(h)$ [18-20], decomposition formulas were obtained for the Appel's hypergeometric functions of two variables by the products of hypergeometric functions of one variable [21]. 
To decompose multiple hypergeometric functions, a multivariable analogue of the above pair of mutually inverse symbolic operators

$$
\tilde{\nabla}_{x_{1} ; x_{2}, \ldots, x_{r}}(h)=\frac{\Gamma(h) \Gamma\left(h+\delta_{1}+\delta_{2}+\ldots+\delta_{r}\right)}{\Gamma\left(h+\delta_{1}\right) \Gamma\left(h+\delta_{2}+\ldots+\delta_{r}\right)}=\sum_{k_{2}, k_{3}, \ldots, k_{r}=0}^{\infty} \frac{\left(-\delta_{1}\right)_{k_{2}+\ldots+k_{r}}\left(-\delta_{2}\right)_{k_{2}} \cdots\left(-\delta_{r}\right)_{k_{r}}}{(h)_{k_{2}+\ldots+k_{r}} k_{2} ! k_{3} ! \cdots k_{r} !}
$$

and

$$
\begin{gathered}
\tilde{\Delta}_{x_{1} ; x_{2}, \ldots, x_{r}}(h)=\frac{\Gamma\left(h+\delta_{1}\right) \Gamma\left(h+\delta_{2}+\ldots+\delta_{r}\right)}{\Gamma(h) \Gamma\left(h+\delta_{1}+\delta_{2}+\ldots+\delta_{r}\right)}= \\
=\sum_{k_{2}, k_{3}, \ldots, k_{r}=0}^{\infty} \frac{\left(-\delta_{1}\right)_{k_{2}+\ldots+k_{r}}\left(-\delta_{2}\right)_{k_{2}} \cdots\left(-\delta_{r}\right)_{k_{r}}}{\left(1-h-\delta_{1}-\ldots-\delta_{r}\right)_{k_{2}+\ldots+k_{r}} k_{2} ! k_{3} ! \cdots k_{r} !}
\end{gathered}
$$

$\left(\delta_{x_{j}}=x_{j} \frac{\partial}{\partial x_{j}} ; j=1,2, \ldots, r\right)$ was introduced in [22].

To study the various properties of another class of generalized multidimensional hypergeometric functions, J. Choi and A. Hasanov [23] introduced the following reciprocal operators:

$$
\begin{gathered}
H_{x_{1}, \ldots x_{r}}(\alpha, \beta)=\frac{\Gamma(\beta) \Gamma\left(\alpha+\delta_{1}+\cdots+\delta_{r}\right)}{\Gamma(\alpha) \Gamma\left(\beta+\delta_{1}+\cdots+\delta_{r}\right)}=\sum_{k_{1}, \cdots, k_{r}=0}^{\infty} \frac{(\beta-\alpha)_{k_{1}+\cdots+k_{r}}\left(-\delta_{1}\right)_{k_{1}} \cdots\left(-\delta_{r}\right)_{k_{r}}}{(\beta)_{k_{1}+\cdots+k_{r}} k_{1} ! \cdots k_{r} !}, \\
\bar{H}_{x_{1}, \ldots x_{r}}(\alpha, \beta)=\frac{\Gamma(\alpha) \Gamma\left(\beta+\delta_{1}+\cdots+\delta_{r}\right)}{\Gamma(\beta) \Gamma\left(\alpha+\delta_{1}+\cdots+\delta_{r}\right)}=\sum_{k_{1}, \ldots k_{r}=0}^{\infty} \frac{(\beta-\alpha)_{k_{1}+\cdots+k_{r}}\left(-\delta_{1}\right)_{k_{1}} \cdots\left(-\delta_{r}\right)_{k_{r}}}{\left(1-\alpha-\delta_{1}-\cdots-\delta_{r}\right)_{i+j} i ! j !} \\
\left(\delta_{x_{j}}=x_{j} \frac{\partial}{\partial x_{j}}(j=1, \ldots, r, r \in \mathbb{N}=\{1,2, \ldots\})\right) .
\end{gathered}
$$

Theorem 1. For the second order hypergeometric functions of four variables (1)-(8), the following operator identities are valid:

$$
\begin{aligned}
& F_{1}^{(4)}\left(a_{1}, a_{2}, b ; c_{1}, c_{2}, c_{3}, c_{4} ; x, y, z, t\right)=H_{t}\left(a_{2}, c_{4}\right)(1-t)^{-b} F_{C}^{(3)}\left(a_{1}, b ; c_{1}, c_{2}, c_{3} ; \frac{x}{1-t}, \frac{y}{1-t}, \frac{z}{1-t}\right), \\
& (1-t)^{-b} F_{C}^{(3)}\left(a_{1}, b ; c_{1}, c_{2}, c_{3} ; \frac{x}{1-t}, \frac{y}{1-t}, \frac{z}{1-t}\right)=\bar{H}_{t}\left(a_{2}, c_{4}\right) F_{1}^{(4)}\left(a_{1}, a_{2}, b ; c_{1}, c_{2}, c_{3}, c_{4} ; x, y, z, t\right),
\end{aligned}
$$

$$
\begin{aligned}
& F_{3}^{(4)}\left(a_{1}, a_{2}, a_{3}, b ; c_{1}, c_{2}, c_{3}, c_{4} ; x, y, z, t\right) \\
& =H_{z}\left(a_{2}, c_{3}\right) H_{t}\left(a_{3}, c_{4}\right)(1-t-z)^{-b} F_{4}\left(a_{1}, b ; c_{1}, c_{2} ; \frac{x}{1-t-z}, \frac{y}{1-t-z}\right), \\
& \begin{array}{r}
(1-t-z)^{-b} F_{4}\left(a_{1}, b ; c_{1}, c_{2} ; \frac{x}{1-t-z}, \frac{y}{1-t-z}\right) \\
=\bar{H}_{z}\left(a_{2}, c_{3}\right) \bar{H}_{t}\left(a_{3}, c_{4}\right) F_{3}^{(4)}\left(a_{1}, a_{2}, a_{3}, b ; c_{1}, c_{2}, c_{3}, c_{4} ; x, y, z, t\right), \\
F_{4}^{(4)}\left(a, b, c ; c_{1}, c_{2}, c_{3}, c_{4} ; x, y, z, t\right)=
\end{array}
\end{aligned}
$$




$$
\begin{gathered}
=H_{z}\left(c, c_{3}\right)(1-z)^{-a} F_{C}^{(3)}\left(a, b ; c_{1}, c_{2}, c_{3} ; \frac{x}{1-z}, \frac{y}{1-z}, \frac{t}{1-z}\right), \\
(1-z)^{-a} F_{C}^{(3)}\left(a, b ; c_{1}, c_{2}, c_{3} ; \frac{x}{1-z}, \frac{y}{1-z}, \frac{t}{1-z}\right) \\
=\bar{H}_{z}\left(c, c_{3}\right) F_{4}^{(4)}\left(a, b, c ; c_{1}, c_{2}, c_{3}, c_{4} ; x, y, z, t\right), \\
F_{5}^{(4)}\left(a_{1}, a_{2}, b_{1}, b_{2} ; c_{1}, c_{2}, c_{3}, c_{4} ; x, y, z, t\right)=H_{t}\left(a_{2}, c_{4}\right)(1-t)^{-b_{2}} F_{E}\left(a_{1} ; b_{1}, b_{2} ; c_{1}, c_{2}, c_{3} ; x, y, \frac{z}{1-t}\right), \\
(1-t)^{-b_{2}} F_{E}\left(a_{1} ; b_{1}, b_{2} ; c_{1}, c_{2}, c_{3} ; x, y, \frac{z}{1-t}\right)=\bar{H}_{t}\left(a_{2}, c_{4}\right) F_{5}^{(4)}\left(a_{1}, a_{2}, b_{1}, b_{2} ; c_{1}, c_{2}, c_{3}, c_{4} ; x, y, z, t\right),
\end{gathered}
$$

$F_{6}^{(4)}\left(a_{1}, a_{2}, b_{1}, b_{2}, b_{3} ; c_{1}, c_{2}, c_{3}, c_{4} ; x, y, z, t\right)$

$$
=H_{y}\left(b_{2}, c_{2}\right) H_{z}\left(b_{3}, c_{3}\right)(1-y-z)^{-a_{1}} F_{2}\left(b_{1} ; a_{1}, a_{2} ; c_{1}, c_{4} ; \frac{x}{1-y-z}, t\right),
$$

$$
\begin{aligned}
& (1-y-z)^{-a_{1}} F_{2}\left(b_{1} ; a_{1}, a_{2} ; c_{1}, c_{4} ; \frac{x}{1-y-z}, t\right) \\
& \quad=\bar{H}_{y}\left(b_{2}, c_{2}\right) \bar{H}_{z}\left(b_{3}, c_{3}\right) F_{6}^{(4)}\left(a_{1}, a_{2}, b_{1}, b_{2}, b_{3} ; c_{1}, c_{2}, c_{3}, c_{4} ; x, y, z, t\right),
\end{aligned}
$$

$F_{8}^{(4)}\left(a_{1}, a_{2}, b_{1}, b_{2} ; c_{1}, c_{2}, c_{3}, c_{4} ; x, y, z, t\right)$

$$
=H_{y}\left(b_{2}, c_{2}\right) H_{t}\left(b_{3}, c_{4}\right)(1-y)^{-a_{1}}(1-t)^{-a_{2}} F_{2}\left(b_{1} ; a_{1}, a_{2} ; c_{1}, c_{3} ; \frac{x}{1-y}, \frac{x}{1-y}\right),
$$

$$
\begin{aligned}
& (1-y)^{-a_{1}}(1-t)^{-a_{2}} F_{2}\left(b_{1} ; a_{1}, a_{2} ; c_{1}, c_{3} ; \frac{x}{1-y}, \frac{x}{1-y}\right) \\
& =\bar{H}_{y}\left(b_{2}, c_{2}\right) \bar{H}_{t}\left(b_{3}, c_{4}\right) F_{8}^{(4)}\left(a_{1}, a_{2}, b_{1}, b_{2} ; c_{1}, c_{2}, c_{3}, c_{4} ; x, y, z, t\right)
\end{aligned}
$$

$F_{11}^{(4)}\left(a_{1}, a_{2}, b ; c_{1}, c_{2}, c_{3} ; x, y, z, t\right)=H_{t}\left(a_{3}, c_{3}\right)(1-t)^{-b} F_{F}\left(b ; a_{1}, a_{2} ; c_{2}, c_{1} ; \frac{y}{1-t}, \frac{z}{1-t}, \frac{x}{1-t}\right)$,

$(1-t)^{-b} F_{F}\left(b ; a_{1}, a_{2} ; c_{2}, c_{1} ; \frac{y}{1-t}, \frac{z}{1-t}, \frac{x}{1-t}\right)=\bar{H}_{t}\left(a_{3}, c_{3}\right) F_{11}^{(4)}\left(a_{1}, a_{2}, b ; c_{1}, c_{2}, c_{3} ; x, y, z, t\right)$,

$$
\begin{aligned}
& F_{13}^{(4)}\left(a_{1}, a_{2}, a_{3}, a_{4}, b ; c_{1}, c_{2}, c_{3} ; x, y, z, t\right) \\
& \quad=H_{z}\left(a_{3}, c_{2}\right) H_{t}\left(a_{4}, c_{3}\right)(1-z-t)^{-b} F_{1}\left(b ; a_{1}, a_{2} ; c_{1} ; \frac{x}{1-z-t}, \frac{y}{1-z-t}\right)
\end{aligned}
$$




$$
\begin{aligned}
(1-z-t)^{-b} F_{1}\left(b ; a_{1}, a_{2} ; c_{1} ;\right. & \left.\frac{x}{1-z-t}, \frac{y}{1-z-t}\right) \\
& =\bar{H}_{z}\left(a_{3}, c_{2}\right) \bar{H}_{t}\left(a_{4}, c_{3}\right) F_{13}^{(4)}\left(a_{1}, a_{2}, a_{3}, a_{4}, b ; c_{1}, c_{2}, c_{3} ; x, y, z, t\right)
\end{aligned}
$$

where $F_{1}, F_{2}, F_{4}$ are Appel hypergeometric functions [21], $F_{C}^{(3)}$ is Lauricella function [24], and $F_{E}, F_{F}$ are Saran functions [25]:

$$
\begin{gathered}
F_{E}\left(\alpha ; \beta_{1}, \beta_{2} ; \gamma_{1}, \gamma_{2}, \gamma_{3} ; x, y, z\right)=\sum_{m, n, p=0}^{\infty} \frac{(\alpha)_{p+m+n}\left(\beta_{1}\right)_{m+n}\left(\beta_{2}\right)_{p}}{\left(\gamma_{1}\right)_{m}\left(\gamma_{2}\right)_{n}\left(\gamma_{3}\right)_{p} m ! n ! p !} x^{m} y^{n} z^{p} \\
F_{F}\left(\alpha ; \beta_{1}, \beta_{2} ; \gamma_{1}, \gamma_{2} ; x, y, z\right)=\sum_{m, n, p=0}^{\infty} \frac{(\alpha)_{m+n+p}\left(\beta_{1}\right)_{m+p}\left(\beta_{2}\right)_{n}}{\left(\gamma_{1}\right)_{m}\left(\gamma_{2}\right)_{n+p} m ! n ! p !} x^{m} y^{n} z^{p}
\end{gathered}
$$

Proof. Theorem 1 is proved by dint of Mellin's transformations [26].

\section{Decomposition formulas}

Theorem 2. For second order hypergeometric functions (1)-(8) the following decomposition formulas are valid:

$$
\begin{aligned}
& F_{1}^{(4)}\left(a_{1}, a_{2}, b ; c_{1}, c_{2}, c_{3}, c_{4} ; x, y, z, t\right) \\
& =(1-t)^{-b} \sum_{i=0}^{\infty} \frac{(-1)^{i}(b)_{i}\left(c_{4}-a_{2}\right)_{i}}{\left(c_{4}\right)_{i} i !}\left(\frac{t}{1-t}\right)^{i} F_{C}^{(3)}\left(a_{1}, b+i ; c_{1}, c_{2}, c_{3} ; \frac{x}{1-t}, \frac{y}{1-t}, \frac{z}{1-t}\right) \\
& (1-t)^{-b} F_{C}^{(3)}\left(a_{1}, b ; c_{1}, c_{2}, c_{3} ; \frac{x}{1-t}, \frac{y}{1-t}, \frac{z}{1-t}\right) \\
& =\sum_{i=0}^{\infty} \frac{\left(c_{4}-a_{2}\right)_{i}(b)_{i}}{\left(c_{4}\right)_{i} i !} t^{i} F_{1}^{(4)}\left(a_{1}, a_{2}, b+i ; c_{1}, c_{2}, c_{3}, c_{4}+i ; x, y, z, t\right),
\end{aligned}
$$

$F_{3}^{(4)}\left(a_{1}, a_{2}, a_{3}, b ; c_{1}, c_{2}, c_{3}, c_{4} ; x, y, z, t\right)$

$$
\begin{aligned}
=(1-t-z)^{-b} & \sum_{i, j=0}^{\infty} \frac{(-1)^{i+j}(b)_{i+j}\left(c_{3}-a_{2}\right)_{i}\left(c_{4}-a_{3}\right)_{j}}{\left(c_{3}\right)_{i}\left(c_{4}\right)_{j} i ! j !}\left(\frac{z}{1-t-z}\right)^{i} \\
& \times\left(\frac{t}{1-t-z}\right)^{j} F_{4}\left(a_{1}, b+i+j ; c_{1}, c_{2} ; \frac{x}{1-t-z}, \frac{y}{1-t-z}\right),
\end{aligned}
$$

$$
\begin{aligned}
& (1-t-z)^{-b} F_{4}\left(a_{1}, b ; c_{1}, c_{2} ; \frac{x}{1-t-z}, \frac{y}{1-t-z}\right) \\
& \quad=\sum_{i, j=0}^{\infty} \frac{\left(c_{3}-a_{2}\right)_{i}\left(c_{4}-a_{3}\right)_{j}(b)_{i+j}}{\left(c_{3}\right)_{i}\left(c_{4}\right)_{j} i ! j !} z^{i} t^{j} F_{3}^{(4)}\left(a_{1}, a_{2}, a_{3}, b+i+j ; c_{1}, c_{2}, c_{3}+i, c_{4}+j ; x, y, z, t\right),
\end{aligned}
$$

$$
\begin{aligned}
F_{4}^{(4)} & \left(a, b, c ; c_{1}, c_{2}, c_{3}, c_{4} ; x, y, z, t\right) \\
& =(1-z)^{-a} \sum_{i=0}^{\infty} \frac{(-1)^{i}(a)_{i}\left(c_{3}-c\right)_{i}}{\left(c_{3}\right)_{i} i !}\left(\frac{z}{1-z}\right)^{i} F_{C}^{(3)}\left(a+i, b ; c_{1}, c_{2}, c_{4} ; \frac{x}{1-z}, \frac{y}{1-z}, \frac{t}{1-z}\right),
\end{aligned}
$$




$$
\begin{aligned}
& (1-z)^{-a} F_{C}^{(3)}\left(a, b ; c_{1}, c_{2}, c_{3} ; \frac{x}{1-z}, \frac{y}{1-z}, \frac{t}{1-z}\right) \\
& \quad=\sum_{i=0}^{\infty} \frac{(a)_{i}\left(c_{3}-c\right)_{i}}{\left(c_{3}\right)_{i} i !} z^{i} F_{4}^{(4)}\left(a+i, b, c ; c_{1}, c_{2}, c_{3}+i, c_{4} ; x, y, z, t\right)
\end{aligned}
$$

$F_{5}^{(4)}\left(a_{1}, a_{2}, b_{1}, b_{2} ; c_{1}, c_{2}, c_{3}, c_{4} ; x, y, z, t\right)$

$$
=(1-t)^{-b_{2}} \sum_{i=0}^{\infty} \frac{(-1)^{i}\left(b_{2}\right)_{i}\left(c_{4}-a_{2}\right)_{i}}{\left(c_{4}\right)_{i} i !}\left(\frac{t}{1-t}\right)^{i} F_{E}\left(a_{1} ; b_{1}, b_{2}+i ; \gamma_{1}, \gamma_{2}, \gamma_{3} ; x, y, \frac{z}{1-t}\right)
$$

$(1-t)^{-b_{2}} F_{E}\left(a_{1} ; b_{1}, b_{2} ; c_{1}, c_{2}, c_{3} ; x, y, \frac{z}{1-t}\right)$

$$
=\sum_{i=0}^{\infty} \frac{\left(b_{2}\right)_{i}\left(c_{4}-a_{2}\right)_{i}}{\left(c_{4}\right)_{i} i !} t^{i} F_{5}^{(4)}\left(a_{1}, a_{2}, b_{1}, b_{2}+i ; c_{1}, c_{2}, c_{3}, c_{4}+i ; x, y, z, t\right)
$$

$F_{6}^{(4)}\left(a_{1}, a_{2}, b_{1}, b_{2}, b_{3} ; c_{1}, c_{2}, c_{3}, c_{4} ; x, y, z, t\right)$

$$
\begin{aligned}
=(1-y-z)^{-a_{1}} \sum_{i, j=0}^{\infty} & \frac{(-1)^{i+j}\left(a_{1}\right)_{i+j}\left(c_{2}-b_{2}\right)_{i}\left(c_{3}-b_{3}\right)_{j}}{\left(c_{2}\right)_{i}\left(c_{3}\right)_{j} i ! j !}\left(\frac{y}{1-y-z}\right)^{i} \\
& \times\left(\frac{z}{1-y-z}\right)^{j} F_{2}\left(b_{1} ; a_{1}+i+j, a_{2} ; c_{1}, c_{4} ; \frac{x}{1-y-z}, t\right), \\
(1-y-z)^{-a_{1}} F_{2}\left(b_{1} ; a_{1}, a_{2} ; c_{1}, c_{4} ;\right. & \left.\frac{x}{1-y-z}, t\right)=\sum_{i, j=0}^{\infty} \frac{\left(a_{1}\right)_{i+j}\left(c_{2}-b_{2}\right)_{i}\left(c_{3}-b_{3}\right)_{j}}{\left(c_{2}\right)_{i}\left(c_{3}\right)_{j} i ! j !} y^{i} z^{j} \\
& \times F_{6}^{(4)}\left(a_{1}+i+j, a_{2}, b_{1}, b_{2}, b_{3} ; c_{1}, c_{2}+i, c_{3}+j, c_{4} ; x, y, z, t\right),
\end{aligned}
$$

$F_{8}^{(4)}\left(a_{1}, a_{2}, b_{1}, b_{2} ; c_{1}, c_{2}, c_{3}, c_{4} ; x, y, z, t\right)=(1-y)^{-a_{1}}(1-t)^{-a_{2}}$

$$
\begin{array}{r}
\times \sum_{i, j=0}^{\infty} \frac{(-1)^{i+j}\left(a_{1}\right)_{i}\left(a_{2}\right)_{j}\left(c_{2}-b_{2}\right)_{i}\left(c_{4}-b_{3}\right)_{j}}{\left(c_{2}\right)_{i}\left(c_{4}\right)_{j} i ! j !}\left(\frac{y}{1-y}\right)^{i}\left(\frac{t}{1-t}\right)^{j} \\
\times F_{2}\left(b_{1} ; a_{1}+i, a_{2}+j ; c_{1}, c_{3} ; \frac{x}{1-y}, \frac{z}{1-y}\right), \\
(1-y)^{-a_{1}}(1-t)^{-a_{2}} F_{2}\left(b_{1} ; a_{1}, a_{2} ; c_{1}, c_{3} ; \frac{x}{1-y}, \frac{x}{1-y}\right)=\sum_{i, j=0}^{\infty} \frac{\left(a_{1}\right)_{i}\left(a_{2}\right)_{j}\left(c_{2}-b_{2}\right)_{i}\left(c_{4}-b_{3}\right)_{j}}{\left(c_{2}\right)_{i}\left(c_{4}\right)_{j} i ! j !} y^{i} t^{j} \\
\times F_{8}^{(4)}\left(a_{1}+i, a_{2}+j, b_{1}, b_{2} ; c_{1}, c_{2}+i, c_{3}, c_{4}+j ; x, y, z, t\right),
\end{array}
$$

$F_{11}^{(4)}\left(a_{1}, a_{2}, b ; c_{1}, c_{2}, c_{3} ; x, y, z, t\right)$

$$
=(1-t)^{-b} \sum_{i=0}^{\infty} \frac{(-1)^{i}(b)_{i}\left(c_{3}-a_{3}\right)_{i}}{\left(c_{3}\right)_{i} i !}\left(\frac{t}{1-t}\right)^{i} F_{F}\left(b+i ; a_{1}, a_{2} ; c_{2}, c_{1} ; \frac{y}{1-t}, \frac{z}{1-t}, \frac{x}{1-t}\right),
$$




$$
\begin{aligned}
& (1-t)^{-b} F_{F}\left(b ; a_{1}, a_{2} ; c_{2}, c_{1} ; \frac{y}{1-t}, \frac{z}{1-t}, \frac{x}{1-t}\right) \\
& =\sum_{i=0}^{\infty} \frac{(b)_{i}\left(c_{3}-a_{3}\right)_{i}}{\left(c_{3}\right)_{i} i !} t^{i} F_{11}^{(4)}\left(a_{1}, a_{2}, b+i ; c_{1}, c_{2}, c_{3}+i ; x, y, z, t\right), \\
& F_{13}^{(4)}\left(a_{1}, a_{2}, a_{3}, a_{4}, b ; c_{1}, c_{2}, c_{3} ; x, y, z, t\right) \\
& =(1-z-t)^{-b} \sum_{i, j=0}^{\infty} \frac{(-1)^{i+j}(b)_{i+j}\left(c_{2}-a_{3}\right)_{i}\left(c_{3}-a_{4}\right)_{j}}{\left(c_{2}\right)_{i}\left(c_{3}\right)_{j} i ! j !}\left(\frac{z}{1-z-t}\right)^{i}\left(\frac{t}{1-z-t}\right)^{j} \\
& (1-z-t)^{-b} F_{1}\left(b ; a_{1}, a_{2} ; c_{1} ; \frac{x}{1-z-t}, \frac{y}{1-z-t}\right) \\
& =\sum_{i=0}^{\infty} \frac{(b)_{i+j}\left(c_{2}-a_{3}\right)_{i}\left(c_{3}-a_{4}\right)_{j}}{\left(c_{2}\right)_{i}\left(c_{3}\right)_{j} i ! j !} z^{i} t^{j} F_{13}^{(4)}\left(a_{1}, a_{2}, a_{3}, a_{4}, b+i+j ; a_{1}, a_{2} ; c_{1} ; \frac{x}{1-z-t}\right)
\end{aligned}
$$

Proof. The proof of Theorem 2 is realized utilizing operator identities (9)-(24), some properties of hypergeometric functions of many variables and the following operator identities [27, p. 93]:

$$
\begin{gathered}
(\delta+\alpha)_{n}\{f(\xi)\}=\xi^{1-\alpha} \frac{d^{n}}{d \xi^{n}}\left\{\xi^{\alpha+n-1} f(\xi)\right\}, \\
(-\delta)_{n}\{f(\xi)\}=(-\xi)^{n} \frac{d^{n}}{d \xi^{n}}\{f(\xi)\}
\end{gathered}
$$

$\delta=\xi \frac{d}{d \xi} ; \alpha \in \mathbb{C} ; n \in \mathbb{N}_{0}=\mathbb{N} \cup\{0\} ; \mathbb{N}=\{1,2,3, \ldots\}$, where $f(\xi)$ is analytical function.

As an example, we give a brief proof of the decomposition (25).

The following equality holds:

$$
(1-t)^{-b} F_{C}^{(3)}\left(a_{1}, b ; c_{1}, c_{2}, c_{3} ; \frac{x}{1-t}, \frac{y}{1-t}, \frac{z}{1-t}\right)=\sum_{m, n, p, q=0}^{\infty} \frac{\left(a_{1}\right)_{m+n+p}(b)_{m+n+p+q}}{\left(c_{1}\right)_{m}\left(c_{2}\right)_{n}\left(c_{3}\right)_{p}} \frac{x^{m}}{m !} \frac{y^{n}}{n !} \frac{z^{p}}{p !} \frac{t^{q}}{q !}
$$

Considering operator definition $H_{t}\left(a_{2}, c_{4}\right)$ and identity (42), from (9) we have

$$
\begin{aligned}
& F_{1}^{(4)}\left(a_{1}, a_{2}, b ; c_{1}, c_{2}, c_{3}, c_{4} ; x, y, z, t\right) \\
& =\sum_{j=0}^{\infty} \frac{\left(c_{4}-a_{2}\right)_{j}\left(-\delta_{t}\right)_{j}}{\left(c_{4}\right)_{j} j !} \sum_{m, n, p, q=0}^{\infty} \frac{\left(a_{1}\right)_{m+n+p}(b)_{m+n+p+q}}{\left(c_{1}\right)_{m}\left(c_{2}\right)_{n}\left(c_{3}\right)_{p}} \frac{x^{m}}{m !} \frac{y^{n}}{n !} \frac{z^{p}}{p !} \frac{t^{q}}{q !} .
\end{aligned}
$$

Using formula (41), we obtain

$$
\begin{aligned}
& F_{1}^{(4)}\left(a_{1}, a_{2}, b ; c_{1}, c_{2}, c_{3}, c_{4} ; x, y, z, t\right) \\
&=\sum_{i=0}^{\infty} \frac{(-1)^{i}(b)_{i}\left(c_{4}-a_{2}\right)_{i}}{\left(c_{4}\right)_{i} i !} t^{i} \sum_{m, n, p, q=0}^{\infty} \frac{\left(a_{1}\right)_{m+n+p}(b+i)_{m+n+p+q}}{\left(c_{1}\right)_{m}\left(c_{2}\right)_{n}\left(c_{3}\right)_{p}} \frac{x^{m}}{m !} \frac{y^{n}}{n !} \frac{z^{p}}{p !} \frac{t^{q}}{q !}
\end{aligned}
$$


By virtue of the validity of the identity $(\lambda)_{m+n}=(\lambda)_{m}(\lambda+m)_{n}$ we get

$$
\begin{aligned}
F_{1}^{(4)}\left(a_{1}, a_{2}, b ; c_{1}, c_{2}, c_{3}, c_{4} ; x, y, z, t\right)= & (1-t)^{-b} \sum_{i=0}^{\infty} \frac{(-1)^{i}(b)_{i}\left(c_{4}-a_{2}\right)_{i}}{\left(c_{4}\right)_{i} i !}\left(\frac{t}{1-t}\right)^{i} \\
& \times \sum_{m, n, p=0}^{\infty} \frac{\left(a_{1}\right)_{m+n+p}(b+i)_{m+n+p}}{\left(c_{1}\right)_{m}\left(c_{2}\right)_{n}\left(c_{3}\right)_{p}} \frac{\left(\frac{x}{1-t}\right)^{m}}{m !} \frac{\left(\frac{y}{1-t}\right)^{n}}{n !} \frac{\frac{z}{1-t} p}{p !} .
\end{aligned}
$$

In view of the $F_{C}^{(3)}$ Lauricella hypergeometric function definition, from expression (43) we obtain decomposition (25).

Thus, the decomposition formula (25) is proved.

Similarly, we can prove each of the decomposition formulas (26)-(40).

Remark 1. The decomposition formulas $(25)-(40)$ can also be proved by comparing the coefficients before the factor $x^{m} y^{n} z^{p} t^{q}$ in both sides of the equality.

\section{Conclusion}

In conclusion, we proved the operator identities written via the mutually inverse operators $H$ and $\bar{H}$ for the hypergeometric functions of four variables $F_{1}^{(4)}, F_{3}^{(4)}-F_{6}^{(4)}, F_{8}^{(4)}, F_{11}^{(4)}, F_{13}^{(4)}$, the validity of the former is proved using the Mellin transforms. By applying the obtained operator identities, differentiation formulas for hypergeometric functions, and properties of hypergeometric functions, we have proved decompositions for the functions $F_{1}^{(4)}, F_{3}^{(4)}-F_{6}^{(4)}, F_{8}^{(4)}, F_{11}^{(4)}, F_{13}^{(4)}$ by products of such known hypergeometric functions as the Appell's functions $F_{1}, F_{2}, F_{4}$; Lauricella's function $F_{C}^{(3)}$; the Saran functions $F_{E}, F_{F}$. Similarly, the decomposition formulas for hypergeometric functions of four variables $F_{17}^{(4)}, F_{18}^{(4)}, F_{19}^{(4)}, F_{20}^{(4)}, F_{21}^{(4)}$, etc. can be obtained.

\section{Acknowledgements}

The study was funded by a grant of the Abai Kazakh National Pedagogical University.

\section{References}

1 Коренев Б.Г. Введение в теорию бесселевых функций / Б.Г. Коренев. - М.: Наука, 1971. — 288 c.

2 Смарт У.М. Небесная механика / У.М. Смарт. - М.: Мир, 1965. - 504 с.

3 Passare M. A multidimensional Jordan residue lemma with an application to Mellin-Barnes integrals / M. Passare, A. Tsikh, O. Zhdanov // Aspects of Mathematics. - 1994. - E 26. P. 233-241. DOI:10.1007/978-3-663-14196-9_8.

4 Candelas P. A pair of Calabi-Yau manifolds as an exactly soluble super conformal theory / P. Candelas, X. de la Ossa, P. Greene, L. Parkes // Nuclear Physics. - 1991. - B 359. P. 21-74. DOI:10.1016/0550-3213(91)90292-6.

5 Varchenko A.N. Multidimensional hypergeometric functions and representation theory of Lie algebras and quantum groups / Advanced Series in Mathematical Physics 21, World Scientific, Singapore, 1995. - $371 \mathrm{p}$.

6 Коваленко А.Д. Теория тонких конических оболочек и ее приложение в машиностроении / А.Д. Коваленко, Я.М. Григоренко, Л.А. Ильин. - Киев: Изд-во АН УССР, 1963. - 287 с. 
7 Виноградов Ю.И. Расчет сферического бака при локальном воздействии / Ю.И. Виноградов, М.В. Константинов // Изв. РАН. Механика твердого тела. - 2016. - № 2. - С. 109-120.

8 Niukkanen A.W. Generalized hypergeometric series ${ }^{N} F\left(x_{1}, \ldots, x_{N} /\right.$ A.W. Niukkanen // Journal of Physics A: Mathematical and General. - 1983. - No. 16. - P. 1813-1825. DOI:10.1088/0305$4470 / 16 / 9 / 007$.

9 Sneddon I.N. Special functions of mathematical physics and chemistry / I.N. Sneddon. - Third Edition, Longman, London, New York, 1980. - 182 p.

10 Srivastava H.M. Special Functions in Queuing Theory and Related Stochastic Processes / H.M. Srivastava, B.R.K. Kashyap. - AP, New York, London, San Francisco, 1982. - 308 p.

11 Srivastava H.M. Double-layer potentials for a generalized bi-axially symmetric Helmholtz equation / H.M. Srivastava, A. Hasanov, J. Choi // Sohag Journal of Mathematics. - 2015. - No. 2 (1). - P. 1-10. DOI:10.12785/sjm/020101.

12 Hasanov A. Fundamental solutions for a class of four-dimensional degenerate elliptic equation / A. Hasanov, A.S. Berdyshev, A. Ryskan // Complex Variables and Elliptic Equations. - 2020. - No. 65(4). - P. 632-647. DOI:10.1080/17476933.2019.1606803.

13 Berdyshev A.S. Double-layer potentials for a generalized bi-axially symmetric Helmholtz equation II / A.S. Berdyshev, A. Hasanov, T. Ergashev // Complex Variables and Elliptic Equations 2020. - No. 65(2). - P. 316-332. DOI:10.1080/17476933.2019.1583219.

14 Exton H. Certain hypergeometric functions of four variables / H. Exton // Bull. Soc. Math. Grece (N.S.). - 1972. - No. 13. - P. 104-113.

15 Sharma C. Hypergeometric functions of four variables (I) / C. Sharma, C.L. Parihar // Journal of Indian Academy of Mathematics. - 1989. - No. 11 (2). - P. 99-1153.

16 Exton H. Some integral representations and transformations of hypergeometric functions of four variables / H. Exton // Bull. Soc. Math. Grece (N.S.). - 1973. - No. 14. - P. 132-140.

17 Exton H. Multiple hypergeometric functions and applications / H. Exton. - John Wilcy and Sons, New York, 1976. - 312 p.

18 Burchnall J.L. Expansions of Appell's double hypergeometric functions Quart / J.L. Burchnall, T.W. Chaundy // J. Math. Oxford Ser. - 1940. - No. 11. - P. 249-270.

19 Burchnall J.L. Expansions of Appell's double hypergeometric functions. II / J.L. Burchnall, T.W. Chaundy // Quart. J. Math. Oxford Ser. - 1941. - 12. - P. 112-128.

20 Chaundy T.W. Expansions of hypergeometric functions / T.W. Chaundy // Quart. J. Math. Oxford Ser. - 1942. - No. 13. - P. 159-171.

21 Erdelyi A. Higher transcendental functions / A. Erdelyi, W. Magnus, Oberhettinger F. Tricomi. - McGraw-Hil, New York, Toronto, London, Vol. I, 1953. - 317 p.

22 Hasanov A. Decomposition formulas associated with the Lauricella multivariable hypergeometric functions / A. Hasanov, H.M. Srivastava // Computers and Mathematics with Applications 2007. - No. 53(7). - P. 1119-1128.

23 Choi J. Applications of the operator $H(\alpha, \beta)$ to the Humbert double hypergeometric functions / J. Choi, A. Hasanov // Computers and Mathematics with Applications. - 2011. - 61. P. 663-671. DOI:10.1016/j.camwa.2010.12.012.

24 Appel P. Fonctions hypergeometriques et hyperspheriques; Polynomes d'Hermite / P. Appel, Kampe de Feriet J. - Gauthier-Villars, Paris, 1926. - 434 p.

25 Saran S. Transformations of certain hypergeometric functions of three variables / S. Saran // Acta Mathematica. - 1955. - 93 (3-4). - P. 292-312.

26 Marichev O.I. Handbook of integral transforms of higher transcendental functions: Theory and algorithmic tables / O.I. Marichev. - Ellis Horwood Ltd, Chichester, 1982. - 336 p. 
27 Poole E. Introduction to the theory of linear differential equations / E. Poole. - Clarendon Press, Oxford, 1936. - 208 p.

\author{
А.С. Бердышев, А. Хасанов, А.Р. Рысқан
}

\title{
Кейбір төрт айнымалы гипергеометриялық қатарлар үшін жіктеу формулалары
}

\begin{abstract}
Мақалада төрт айнымалы гипергеометриялық Гаусс қатарлары үшін операторлық тепе-теңдік пен қарапайым функцияларға жіктеу формулалары алынды. Символдық операторлардың кері жұптарына негізделген Чои-Хасанов әдісі қолданылды. Алынған төрт айнымалы гипергеометриялық қатарлары үшін жіктеу формулалары осы функциялардың қасиеттерін зерттеп білуге мүмкіндік береді. Алынған жіктеулер көпөлшемді азғындалған дербес туындылы дифференциалдық теңдеулер үшін шеттік есептердің шешілімділік мәселелерін зерттеуде пайданылады.
\end{abstract}

Кілт сөздер: Аппель гипергеометриялық функциясы, Лауричелл функциясы, Саран функциясы, төрт айнымалы гипергеометриялық қатар, жіктеу формулалары, операторлық тепе-теңдіктер, кері символдық операторлар.

\author{
А.С. Бердышев, А. Хасанов, А.Р. Рыскан \\ Формулы разложения для некоторых \\ гипергеометрических рядов четырех переменных
}

В статье получены операторные тождества и формулы разложения для гипергеометрических рядов Гаусса второго порядка четырех переменных по произведениям, содержащим более простые гипергеометрические функции. Авторами использован метод Чои-Хасанова, основанный на обратных парах символических операторов. Полученные формулы разложения для гипергеометрических функций четырех переменных позволят изучить свойства этих функций. Данные разложения применяются при исследовании вопросов разрешимости краевых задач для вырождающихся многомерных дифференциальных уравнений в частных производных.

Ключевые слова: гипергеометрическая функция Аппеля, функция Лауричелла, функция Сарана, гипергеометрический ряд четырех переменных, формулы разложения, операторные тождества, обратные символические операторы.

\section{References}

1 Korenev, B.G. (1971). Vvedenie v teoriiu besselevykh funktsii [Introduction to the theory of Bessel functions]. Moscow: Nauka [in Russian].

2 Smart, U.M. (1965). Nebesnaia mekhanika /Celestial mechanics]. Moscow: Mir [in Russian].

3 Passare, M., Tsikh, A. \& Zhdanov, O. (1994). A multidimensional Jordan residue lemma with an application to Mellin-Barnes integrals. Aspects of Mathematics, E 26, 233-241. DOI:10.1007/9783-663-14196-9_8.

4 Candelas, P., de la Ossa, X., Greene, P. \& Parkes, L. (1991). A pair of Calabi-Yau manifolds as an exactly soluble super conformal theory. Nuclear Physics, B 359, 21-74. DOI:10.1016/05503213(91)90292-6.

5 Varchenko, A.N. (1995). Multidimensional hypergeometric functions and representation theory of Lie algebras and quantum groups. Singapore: Advanced Series in Mathematical Physics, 21, World Scientific. 
6 Kovalenko, A.D., Grigorenko, Ya.M. \& Ilyin, L.A. (1963). Teoriya tonkikh konicheskikh obolochek $i$ yeyo prilozheniya $v$ mashinostroyenii /The theory of thin conical shells and its application in mechanical engineering]. Kiev: AS USSR [in Russian].

7 Vinogradov, Yu.I. \& Konstantinov, M.V. (2016). Raschet sfericheskoho baka pri lokalnom vozdeistvii [Calculation of a spherical tank under local exposure]. Izvestiia RAN. Mekhanika tverdoho tela - Bulletin of the RAS. Mechanics of Solids, Vol. 2, 109-120 [in Russian].

8 Niukkanen, A.W. (1983). Generalized hypergeometric series ${ }^{N} F\left(x_{1}, \ldots, x_{N}\right.$. Journal of Physics A: Mathematical and General, 16, 1813-1825. DOI:10.1088/0305-4470/16/9/007.

9 Sneddon, I.N. (1980). Special functions of mathematical physics and chemistry / Third Edition, Longman, London, New York.

10 Srivastava, H.M., \& Kashyap, B.R.K. (1982). Special Functions in Queuing Theory and Related Stochastic Processes AP, New York, London, San Francisco.

11 Srivastava, H.M., Hasanov, A. \& Choi, J. (2015). Double-layer potentials for a generalized biaxially symmetric Helmholtz equation. Sohag Journal of Mathematics, 2(1), 1-10. DOI: 10.12785 $/ \operatorname{sjm} / 020101$.

12 Hasanov, A., Berdyshev, A.S. \& Ryskan, A. (2020). Fundamental solutions for a class of fourdimensional degenerate elliptic equation. Complex Variables and Elliptic Equations, 65(4), 632647. DOI:10.1080/17476933.2019.1606803.

13 Berdyshev, A.S., Hasanov, A. \& Ergashev, T. (2020). Double-layer potentials for a generalized bi-axially symmetric Helmholtz equation II. Complex Variables and Elliptic Equations, 65 (2), 316-332. DOI:10.1080/17476933.2019.1583219.

14 Exton, H. (1972). Certain hypergeometric functions of four variables. Bull. Soc. Math. Grece (N.S.), 13, 104-113.

15 Sharma, C. \& Parihar, C.L. (1989). Hypergeometric functions of four variables (I). Journal of Indian Academy of Mathematics, 11(2), 99-1153.

16 Exton, H. (1973). Some integral representations and transformations of hypergeometric functions of four variables. Bull. Soc. Math. Grece (N.S.), 14, 132-140.

17 Exton, H. (1976). Multiple hypergeometric functions and applications John Wilcy and Sons, New York.

18 Burchnall, J.L. \& Chaundy, T.W. (1940). Expansions of Appell's double hypergeometric functions Quart. J. Math. Oxford Ser. , 11, 249-270.

19 Burchnall, J.L. \& Chaundy, T.W. (1941). Expansions of Appell's double hypergeometric functions. II Quart. J. Math. Oxford Ser. , 12, 112-128.

20 Chaundy, T.W. (1942). Expansions of hypergeometric functions Quart. J. Math. Oxford Ser., 13, 159-171.

21 Erdelyi A., Magnus W., Oberhettinger, F. \& Tricomi F.G. (1953).Higher transcendental functions, Vol. I McGraw-Hil, New York, Toronto, London.

22 Hasanov, A. \& Srivastava, H.M. (2007). Decomposition formulas associated with the Lauricella multivariable hypergeometric functions Computers and Mathematics with Applications, 53 (7), 1119-1128.

23 Choi, J. \& Hasanov, A. (2011). Applications of the operator $H(\alpha, \beta)$ to the Humbert double hypergeometric functions Computers and Mathematics with Applications, 61, 663-671. DOI: 10.1016/j.camwa.2010.12.012.

24 Appell, P. \& Kampe de Feriet, J. (1926).Fonctions hypergeometriques et hyperspheriques; Polynomes d'Hermite Gauthier-Villars, Paris. 
25 Saran, S. (1955). Transformations of certain hypergeometric functions of three variables Acta Mathematica, 93 (3-4), 292-312.

26 Marichev, O.I. (1982). Handbook of integral transforms of higher transcendental functions: Theory and algorithmic tables Ellis Horwood Ltd, Chichester.

27 Poole, E. (1936). Introduction to the theory of linear differential equations Clarendon Press, Oxford. 